\title{
1 Droplet Size and Velocity Distributions of Wave-Impact Sea Spray over a Marine Vessel
}

3

\author{
S.R. Dehghani ${ }^{1}$, G.F. Naterer, Y.S. Muzychka \\ Department of Mechanical Engineering, Faculty of Engineering and Applied Science, \\ Memorial University of Newfoundland, St. John’s, NL, A1B 3X5, Canada
}

\section{Abstract}

The spatial distribution of droplets in a spray cloud created by wave-impact sea spray and the distribution of their sizes and velocities over a vessel deck is investigated. Wave-impact sea spray, which occurs due to striking high energy sea waves on a vessel's bow, creates numerous droplets in front of a vessel. Droplets are frequently the result of sheet and droplet breakup of sea water. The velocity-size dependence of the resultant droplets is important in the modelling of marine icing phenomena. A droplet trajectory method employs the velocity-size dependence of the droplets to find their spatial distributions in the cloud of spray over the vessel deck. Drag body forces overcome the initial velocities of the droplets so they follow the wind direction and gravitational direction. The motion of the droplets affects the shape and extent of the spray cloud in front of the vessel and over the deck. In this paper, numerical methods are developed to find the distribution of sizes and velocities of the droplets over a vessel. Results show that neither the smallest nor the largest droplets reach the maximum height. The medium-size droplets can reach the maximum height of the spray cloud. As the spray cloud travels over the deck, the droplet velocities become almost the same. Comparing the numerical results with field observations shows

\footnotetext{
${ }^{1}$ Corresponding author, Postdoctoral Fellow, Phone: +1 (709) 771-6216, Email: srdehghani@mun.ca
} 
23 that the predicted results are consistent and have reasonable agreement with the field 24 measurements.

26 Keywords: Droplet size distribution, Droplet velocity distribution, Marine icing, Wave-impact 27 sea spray, Droplet trajectory

\section{Introduction}

30 Wave-impact sea spray, which results from high energy sea waves striking a vessel bow or hull,

31 is the main reason for marine icing in cold regions (Zakrzewski, 1987; Lozowski et al., 2000;

32 Panov, 1978). Every spray cloud carries numerous droplets towards the vessel platform

33 (Zakrzewski, 1986; Zakrzewski and Lozowski, 1988). The nature of the spray cloud and droplets

34 affects the progress of ice accretion on a marine vessel (Ryerson, 1995; Borisenkov et al., 1975).

35 A spray cloud can be defined based on time dependent spatial distributions of sizes and velocities

36 of the droplets. The spatial distributions, including the velocity and size of the droplets, determine

37 the spray cloud (Zakrzewski and Lozowski, 1988; Dehghani et al., 2016a).

Apart from the ambient temperature, relative humidity and wind velocity, the incoming

39 water flux to a vessel deck is important for calculating the amount of accumulated ice on the vessel

40 (Kulyakhtin and Tsarau, 2014; Horjen, 2013). The accumulated ice is brine-spongy ice (Dehghani

41 et al., 2016b). The water flux varies with position and time. Size and velocity distributions of the

42 droplets in a spray cloud determine the local spray flux at every point of a vessel. Distributions of

43 size and velocity will yield the water flux, which will also be a function of time and space

44 (Dehghani et al., 2016a).

Past studies reported mono-size models where there is no distribution of size and velocity

46 for a cloud of spray (Kulyakhtin and Tsarau, 2014). Horjen (2013) used a size of $1.8 \mathrm{~mm}$ for the 
47 droplets. Shipilova et al. (2012) assumed $0.25 \mathrm{~mm}$ and $2 \mathrm{~mm}$ as the droplet sizes. Horjen (2015)

48 considered the size of the droplets as $3.8 \mathrm{~mm}$. Chung and Lozowski (1998) assumed the same size

49 of droplets as Zakrzewski (1986), $1.75 \mathrm{~mm}$. Kulyakhtin and Tsarau (2014) mentioned that droplet

50 sizes are between 1 and $2 \mathrm{~mm}$. These past studies assumed the initial velocity of the droplets as

51 equal to the wind velocity. A lack of distribution of size and velocity in a spray cloud led the

52 researchers to use mono-size and mono-velocity models.

Droplet trajectory modes can predict the droplet paths and consequently their positions.

54 When a spray cloud moves, droplets start their movements at their initial positions and finish by

55 impinging on vessel surfaces (Dehghani et al., 2009; Zakrzewski and Lozowski, 1988). The droplet

56 trajectory method, which needs the initial size and velocity distributions, will determine the

57 distribution of the size and velocity of the droplets at every section of the spray cloud, and consequently, the final distribution of the spray flux over the vessel surfaces (Dehghani et al., 59 2013; Dehghani et al., 2016a).

The liquid water content (LWC) of a spray cloud over a vessel deck ise affected by the

61 distributions of size and velocity of droplets over a vessel platform (Ryerson, 1995; Dehghani et

62 al., 2016a). The collision efficiency, which is a key parameter in calculating the fraction of 63 impingement of the droplets on a specific surface, is also a function of the size and velocity of the 64 droplets close to the surface (Zakrzewski, 1986). The freezing rate can be affected by the incoming 65 flux of water and the collision efficiency. Both are also dependent on the distribution of size and 66 velocity of droplets (Chung et al., 1998a; Chung et al., 1998b; Sharpov, 1971; Shipilova, et al. 67 2012).

Therefore, determination of the distributions of size and velocity of the droplets and their 69 variations over marine vessels during the motion of a spray cloud are essential for accurate 
modelling of marine icing phenomena (Zakrzewski and Lozowski, 1988). The assumptions of

71 constant droplet sizes and velocities used in previous models of marine icing are not satisfactory.

72 This assumption does not yield a sufficiently accurate estimation of ice accretion over a marine

73 vessel. A vertically uniform size and velocity are the most common assumption in past studies

74 (Horjen, 2013; Horjen, 2015; Kulyakhtin and Tsarau, 2014; Lozowski et al., 2000; Shipilova, et 75 al., 2012).

This paper focuses on new models for the distributions of size and velocity of droplets in

77 a spray cloud over a marine vessel, using a droplet trajectory method and droplet-size-dependent

78 characteristics after water breakup in front of a vessel. A new distribution of size and velocity is

79 presented. The distribution of size and velocity can determine the extent of the spray cloud over a

80 vessel. The model will be examined and compared against data from field observations.

81

\section{2. Spray Cloud Processes}

83 Wave-impact sea spray is created by high energy sea waves striking a vessel bow or hull (Dehghani

84 et al., 2016a; Zakrzewski, 1986). The process of creating a spray cloud and its development and

85 motion can be divided into several stages: wave impact, sheet breakup, droplet breakup, spray

86 cloud formation, spray cloud acceleration and deceleration, and spray cloud fall and impingement.

87 These stages have not been well understood (Dehghani et al., 2016a; Ryerson 1995). The mechanism of sheet creation, sheet breakup and droplet breakup have been examined in a few past

89 studies but need more investigation (Bullock et al., 2007; Galiev and Flay, 2014; Greco et al., 90 2013; Gu et al., 2014; Ren and Marshall, 2014; Dehghani et al., 2016a).

After the stage of droplet breakup, there are numerous droplets with various sizes and

92 velocities in the spray cloud. At the front edge of the vessel, the droplets are moving upward and 
93 in the same direction as the vessel. The stage of spray cloud formation begins with decelerating 94 and accelerating droplets. Upward movement of the droplets is decelerated by drag forces and 95 body forces. Drag forces are created as a result of the relative velocity of the droplets and wind. 96 Body forces occur with the gravity force exerted on the droplets. Due to the drag force and body 97 force, the vertical component of droplet velocities decreases to reach zero. At this point, droplets 98 reach their maximum height. The horizontal components of the droplet velocities experience the 99 same trend. The start of the horizontal movement of the droplets is usually in the opposite direction 100 of the wind velocity. The wind slows down the droplets. After a short period, in the decelerating 101 period, the horizontal velocities of the droplets become zero. This point is the maximum horizontal 102 development of a spray cloud in the opposite direction of the wind.

103 Droplets with a vertical velocity of zero, which are at their maximum heights, start 104 downward movement because of gravity. This accelerates the droplets to reach their terminal 105 velocities. The droplets with zero horizontal velocities are affected by the wind velocity and 106 increase their velocities. The wind accelerates these droplets and increases their velocities to the 107 wind velocity. Accelerating the droplets is continued until the droplets impinge on the vessel 108 surfaces.

The spray cloud fall and droplet impingement are the last stages of motion of a spray cloud 110 over a marine vessel. The various droplets with various sizes and velocities take different paths 111 and reach different positions. The drag force, wind velocity, droplet size, and initial velocity of 112 droplets determine the trajectory of the droplets. Figure 1 illustrates these stages of a spray cloud 113 development related to wave-impact sea spray over a marine vessel. The vessel chosen is the same 114 as a Medium-size Fishing Vessel (MFV) (Borizenkov et al., 1975; Zakrzewski, 1986; Sharpov, 115 1971). The important components of the MFV are illustrated in the figure. The overall length of 
116 the vessel is about $39.5 \mathrm{~m}$. The foremast is located at $11.0 \mathrm{~m}$ from the ship bow. The front side of

117 the structure is located at a distance of 19.2 from the ship bow. The height of the structure above

118 the deck is $4.5 \mathrm{~m}$. The life boat is located 29.0 to $34.1 \mathrm{~m}$ from the ship bow (Zakrzewski and

119 Lozowski, 1988).

The initial velocities and sizes of droplets at the front edge of a marine vessel are among

121 the most essential elements for predicting the droplet trajectories. A velocity-size dependence

122 suggests that after the droplet breakup stage, the larger droplets have lower velocities and the

123 smaller droplets have higher velocities. This means at the front edge of the vessel, there is a 124 velocity-size dependence for the droplets that can be used for the initial conditions. Dehghani et 125 al. (2016a) reported this velocity-size dependence.

126

127

\section{Formulation of Spray Cloud Motion}

Spherical droplets, having a density of $\rho_{d}$ and a diameter of $D_{d}$, are small compared to the and substituting the body force and drag force will result in the following equation of the droplet trajectory. The equation describing to droplet movement and the forces acting on them can be

132 expressed as:

$$
m_{d} \frac{d \boldsymbol{V}_{\boldsymbol{d}}}{d t}=\rho_{d} \forall_{d} \mathbf{g}-C_{d r} \frac{\pi D_{d}^{2}}{8} \rho_{a}\left|\boldsymbol{V}_{\boldsymbol{d}}-\boldsymbol{V}_{\boldsymbol{a}}\right|\left(\boldsymbol{V}_{\boldsymbol{d}}-\boldsymbol{V}_{\boldsymbol{a}}\right)+\rho_{a} \forall_{d} C_{a d} \frac{D\left(\boldsymbol{V}_{\boldsymbol{a}}-\boldsymbol{V}_{\boldsymbol{d}}\right)}{D t}+\rho_{a} \forall_{d}\left(\frac{D \boldsymbol{V}_{\boldsymbol{a}}}{D t}-\right.
$$

135 where $m_{d}$ is mass of the droplet, $t$ is time, $\boldsymbol{V}_{\boldsymbol{d}}$ is droplet velocity, $\boldsymbol{V}_{\boldsymbol{a}}$ is air velocity, $\rho_{\boldsymbol{d}}$ is water density, $\forall_{d}$ is droplet volume, $\mathbf{g}$ is gravity, $C_{d r}$ is drag coefficient, $D_{d}$ is droplet diameter, $\rho_{a}$ is air

137 density, and $C_{a d}$ is added mass force coefficient. The coefficient $C_{a d}$ is assumed to be 0.5 and $138 C_{d r}$ can be calculated as follows (Dehghani et al. 2009): 


$$
C_{d r}=\left\{\begin{array}{lc}
\frac{24}{R e} & R e<1 \\
\frac{24}{R e}\left(1+0.15 R e^{0.687}\right) & 1<R e<1000 \\
0.44 & R e>1000
\end{array}\right.
$$

In order to solve this equation, its unknowns are calculated separately. Substituting the

141

142

143

144

145

146

147

148

149

150

151

152

153

154

155

156

157 158

159

unknowns leads to a set of ordinary differential equations as follows.

$$
\begin{aligned}
& \dot{x}=\frac{d x}{d t}, \quad \ddot{x}=\frac{d^{2} x}{d t^{2}}, \quad \ddot{x}=-\frac{3 C_{d r}}{4 D\left(\gamma+C_{a d}\right)}(\dot{x}-U) \sqrt{(\dot{x}-U)^{2}+\dot{z}^{2}} \\
& \dot{z}=\frac{d z}{d t}, \quad \ddot{z}=\frac{d^{2} z}{d t^{2}}, \quad \ddot{z}=\left(\frac{1-\gamma}{\gamma+C_{a d}}\right) \mathrm{g}-\frac{3 C_{d r}}{4 D\left(\gamma+C_{a d}\right)}(\dot{z}) \sqrt{(\dot{x}-U)^{2}+\dot{z}^{2}}
\end{aligned}
$$

where $x, \dot{x}, \ddot{x}, z, \dot{z}$, and $\ddot{z}$, are position, velocity, and acceleration of the droplets, $\gamma$ is the liquid density to air density ratio, and $U$ is the relative velocity of wind to the vessel. The initial conditions are droplet sizes and velocities. This set of six equations and six unknowns is solved with a standard numerical solver.

There are various suggested formulae for LWC due to wave-impact sea spray, but many are intended for offshore structurers (Forest et al., 2005). The field observations of Borisenkov et al. (1975) are the most relevant data that can be used in this instance. These data related to the MFV which are suitable for our model and can be used to examine the droplet trajectory results. The proposed relation that represents the liquid water content is given by:

$$
w=24.2 \times \exp (-0.55 z) \quad g r_{\text {water }} / \mathrm{m}^{3} \text { air }
$$

where $z$ is the elevation above the deck of the MFV (Zakrzewski, 1987) and $w$ is the LWC.

\section{Numerical Results}

The spray cloud motion can be quantified using the previous droplet trajectory model. Initial sizes of the droplets are chosen based on past work by Ryerson (1995), who reported the droplet sizes in a range from close to zero to $7.7 \mathrm{~mm}$. Therefore, the initial distribution of sizes contains droplet 
diameters from zero to $7 \mathrm{~mm}$. The initial velocity distribution is chosen by considering the velocity-size dependence of the droplets at the end of water breakup. In this case, the maximum initial velocity is considered as $60 \mathrm{~m} / \mathrm{s}$. Therefore, there is a distribution of size and velocity that can be used as the initial condition at the front edge of the vessel. The model assumes that the wind velocity is uniform, horizontal and equal to $11 \mathrm{~m} / \mathrm{s}$, which is equal to the wind velocity of the MFV on the Sea of Japan as reported by Borisenkov et al. (1975). The heading angle is considered as $180^{\circ}$. The ship velocity is assumed to be $2.83 \mathrm{~m} / \mathrm{s}$, which is equal to the MFV velocity on the Sea of Japan as reported by Borisenkov et al. (1975). The mass fraction of evaporation is assumed negligible. The spray is assumed dilute; therefore, the droplets will not affect each other and the droplet trajectory can be used for every droplet individually. The breakup is assumed to be finished at the vessel edge in front of the vessel.

The extent of the spray cloud is the first important parameter in the marine icing analysis. A high spray cloud can cause the creation of ice on the high elevations of the vessel. The accumulated ice on the high elevations changes the center of mass of the vessel to a higher level. This phenomenon causes an instability of the vessel that increases the risk of capsize. Therefore, the height of spray is an important factor in the modelling of marine icing phenomena. Figure 2 shows the results of the numerical solution, which are attached to the vessel sketch. The droplet trajectory method results in the creation of droplet paths over the deck. The dashed lines represent droplet paths over the vessel deck. The model calculates a full distribution of sizes and velocities as mentioned before. Figure 2 shows the trajectories of some droplets to represent the spray cloud. The spray cloud impinges on the foremast and the front side of the structure. Therefore ice accretion on these surfaces is expected. The spray cloud cannot reach the roof of the structure and the other areas that are far from the front of the vessel. 
The largest and smallest droplets cannot reach the highest positions. Figure 2 shows that

$6.6 \mathrm{~mm}$ droplets fall to the deck very quickly. Their maximum heights are less than $0.2 \mathrm{~m}$ and their maximum ranges are less than $0.6 \mathrm{~m}$. The maximum height for the droplets with a $0.3 \mathrm{~mm}$ diameter is about $1.5 \mathrm{~m}$. The maximum height occurs for the medium-sized droplets. The droplets with 2.4 to $3.8 \mathrm{~mm}$ diameter can reach the maximum height which is about $8 \mathrm{~m}$ and is located between the front edge of the vessel and the foremast.

The smaller droplets are rapidly affected by the wind. They are light and the wind can carry them more easily than heavy droplets. A competition between drag forces and the body forces determines the paths of the droplets. Larger droplets are heavy and lower velocity, while smaller droplets are light and higher velocity. Higher velocity droplets imply higher drag forces. Therefore, medium-size droplets are faced with values of body and drag forces that let them travel to the maximum height. The small and large droplets cannot reach the highest height because of their drag forces and body forces respectively.

Analyzing the vertical distribution of droplet sizes can clarify the extent of motion of the spray cloud. Figure 3 shows the size distribution of the droplets in the spray cloud in five cross sections. At $x=0$, which occurs at the front edge of the vessel, the droplet size distribution includes droplets with sizes from $7 \mathrm{~mm}$ to very small droplets. The maximum height in this section is about $6 \mathrm{~m}$. The larger and smaller droplets are at lower heights. The medium-size droplets can reach the high heights. At $x=5 \mathrm{~m}$, the maximum height of the spray cloud occurs. The maximum height is about $8 \mathrm{~m}$. There are no droplets larger than $6 \mathrm{~mm}$ in this section. This means that 6 to 7 mm droplets fall to the deck between $x=0$ and $x=5 \mathrm{~m}$. The next section, $x=10 \mathrm{~m}$, includes the droplet sizes smaller than $5.3 \mathrm{~mm}$. The maximum height is less than $7 \mathrm{~m}$. For the last section, the droplet sizes are limited to less than $4.6 \mathrm{~mm}$. This means the larger droplets, which are heavier, 
fall to the deck before reaching this section. As with the other sections, the larger and smaller droplets are at lower heights and the medium-size droplets can reach higher heights.

Vertical distributions of the droplet velocities are further important factors. Figure 4 shows the vertical distribution of the horizontal component of the velocity of the droplets in the five sections. In the first section, at $x=0$, the distribution is completely different than the other sections. This section is located at the acceleration stage and the droplets are accelerated by the wind. The droplets are at the minimum horizontal velocities. The wind velocity will affect the droplets and carry them. The horizontal velocity of the droplets is expected to increase. In the next sections, the horizontal velocity increases. Figure 4 shows that the maximum velocity will be less than $14 \mathrm{~m} / \mathrm{s}$, which is very close to the relative velocity of the vessel and wind. The small droplets will have the same velocity as the wind after the second section. The difference between the horizontal velocities in each section decreases as $x$ increases. This means the droplets tend to reach a uniform horizontal velocity as they travel over the deck.

The vertical distributions of the vertical components of the velocities of the droplets vary as the spray cloud travels. As with the horizontal velocity, the distribution of the vertical velocities in the section of $x=0$ is different. Droplets are decelerated to a zero velocity. In this section, the droplets move upward and the vertical velocity is positive. The maximum vertical velocity is less than $11 \mathrm{~m} / \mathrm{s}$. In the other sections, the droplets are falling. The minimum velocity is about $8.2 \mathrm{~m} / \mathrm{s}$. The differences between the vertical velocities of the droplets increase as they travel over the deck. They are affected by the forces, drag force and body force, in different ways.

The distributions of the total velocity of the droplets are shown in Figure 6. The total velocities vary between 0.4 and $14 \mathrm{~m} / \mathrm{s}$. For $x=0$, the droplet velocities vary between 0.4 and $12.4 \mathrm{~m} / \mathrm{s}$. This is the widest range of the velocities. Some droplets, the largest, have the lowest 
229 velocity and some droplets, the smallest, have the highest velocity. The tightest range occurs for

$230 x=15 \mathrm{~m}$. In this section, the velocities of the droplets are about $14 \mathrm{~m} / \mathrm{s}$. As the spray cloud travels

231 over the deck, the differences between the velocities decreases. The droplets correct their velocities

232 and reach almost the same velocities after a short time.

As the spray cloud travels over the deck, large and low velocity droplets fall to the deck.

234 Therefore, the LWC is expected to decrease. Figure 7 shows the variation of the LWC over the

235 MFV at various $x$ distances to the front edge of the vessel. The maximum amount of LWC occurs

236 at $x=0$, which is at the front of the vessel. At this point the variation of the LWC vs. height is

237 approximately exponential. At $x=5 \mathrm{~m}$, the height of the spray increases but LWC decreases and

238 the curve fluctuates between 3 and $4 \mathrm{gr} / \mathrm{m}^{3}$. At $x=10 \mathrm{~m}$, the height of the spray decreases and

239 the LWC decreases as well. The LWC is about $1 \mathrm{gr} / \mathrm{m}^{3}$ at $x=20 \mathrm{~m}$. This position is close to the

240 front side of the structure of the vessel.

241 Droplet movements can be forward, which means co-flowing with the wind velocity,

242 backward, which is a counter-direction with the wind velocity, upward, which is against gravity,

243 and downward. The travel angle can define the type of movement. The angles between zero and

$24490^{\circ}$ mean forward-upward directions and the angles between zero and $-90^{\circ}$ mean forward-

245 downward directions. Figure 1 illustrates the definition of the travelling angle, $\theta$. Figure 8 shows

246 the distributions of the traveling angles at various sections. At $x=0$, the droplets are moving in

247 forward-upward directions. This means the droplets are travelling towards the vessel and also

248 towards the higher heights. All the droplets at $x=5 \mathrm{~m}$ are travelling downward. This means they

249 are in the stage of descent. As the spray cloud travels over the deck, the medium-size droplets

250 move increasingly downward. This means the medium-size droplets are the last droplets that are

251 affected by gravity. 
Analyzing the distribution of size and velocity of the droplets can show that at the 253 acceleration and deceleration stages, the droplets are expanding the spray cloud. After a full 254 expansion, they start falling. The wind velocity affects the droplets in different ways. The smaller 255 droplets are carried by wind and the larger droplets impinge on the deck rapidly.

257 movement of the droplets in the air stream. The horizontal component of the drag force is a 258 resistance force in the direction of the wind velocity. The maximum resistance occurs at the start 259 of the development of the spray cloud when droplets are injected into the wind stream in the 260 opposite direction. The drag force reduces the droplet velocities and decelerates them. The 261 acceleration stage is started when droplets reach their minimum velocities. The droplets are 262 accelerated and their velocities are increased over the deck. Figure 9 shows the distributions of the 263 horizontal components of the drag forces of the droplets over the MFV. As the figure shows, the 264 resistance force is higher at $x=0$, especially for the small and high velocity droplets. This causes 265 the droplets to reduce their velocities. At $x=5 \mathrm{~m}$, the drag force decreases, the droplets are 266 accelerated, and their velocities become close to the wind velocity. At $x=10,15$, and $20 \mathrm{~m}$, the 267 situations are the same. The droplet velocities are closer to the wind velocity and the drag forces 268 decrease. Figure 9 shows that the horizontal drag forces occur in the same direction as the wind 269 velocity. This means the drag force helps droplets to be aligned with the wind throughout the 270 process of the spray cloud development.

271 The vertical components of the drag forces affect the vertical movements of the droplets.

272 At the start of the spray cloud formation, the vertical drag force is downward. This reduces the 273 droplet velocities and prevents their further upward movement. The droplet velocities reach zero 274 and then start falling down. At $x=0$, the drag forces are negative and in the other sections the 
275 drag forces are positive. At the start of the formation of the spray cloud, the vertical components

276 of drag forces tend to reduce the upward velocities of the droplets. In the other sections, the

277 droplets are falling down and the drag forces tend to resist their fall.

278 Figure 11 shows the distributions of the body forces in five sections over the deck of the

279 MFV. The larger droplets have the higher body forces. The balance of the body force and the

280 vertical component of the drag force determine the vertical movement of the droplets in the cloud

281 of spray. The maximum body force occurs for the largest droplets, which are located at $x=0$. As

282 the spray cloud moves ahead, the large droplets fall down and the maximum value of the body

283 forces reduces. Comparing Figs. 10 and 11 shows that as the spray cloud moves over the deck, the

284 differences between the drag forces and body forces decrease. This shows that the droplets reach

285 their terminal velocities in the last sections.

286

Figure 12 shows the distributions of the total drag forces in various cross-sections over the

287 MFV. The maximum drag force occurs for the high velocity droplets at $x=0$. These droplets that

288 are the smallest sizes reduce their velocities in a short period. The drag force for the largest droplets

289 is not the minimum drag in this section. The largest droplets have the smallest velocities and drag

290 forces. The medium-size droplets that have a medium velocity and size have a moderate drag force.

291 They are not heavy enough to be affected by gravity and they are not fast enough to be stopped by

292 the drag force. This explains why they can reach the maximum height in the spray cloud. For the

293 other sections, the drag forces decrease because of the lower relative velocities. The small droplets

294 that have the same velocity as the wind have small drag forces.

295

The effect of the spray cloud on some parts of the MFV has been reported by Sharpov

296 (1971). Table 1 shows a comparison between the results of the present model, observations of

297 Sharpov (1971), and the results of Zakrazowski and Lozowski (1988). As shown in the table, the 
298 wet height of the foremast, which is the minimum height of the foremast hit by the spray cloud, is 299 predicted as about $6.28 \mathrm{~m}$. In this situation, the prediction of Zakrazowski and Lozowski (1988) is about $5.85 \mathrm{~m}$ and the observation of Sharpov (1971) is between 5.9 and $7.9 \mathrm{~m}$. The model predicts 301 that the front side of the structure becomes wet. The wet height is about $2.33 \mathrm{~m}$. The result of 302 Zakrazowski and Lozowski (1988) is $2.07 \mathrm{~m}$ and Sharpov (1971) does not mention the wet height, 303 but mentions that the spray hits the front side of the superstructure. The spray cloud cannot wet 304 the other parts such as the roof of the structure, the boat deck, and the safety boat. The model, 305 predictions and the observations are in a reasonable agreement. and the LWC obtained by the numerical model. The numerical results are well aligned with the measured results. The exponential form of the fitted curve of the observations is in useful agreement with the numerical results. The LWC corresponds to the section of $x=0$, which is the 310 front edge of the vessel. The maximum height of the spray at $x=0$ is about $6 \mathrm{~m}$. Therefore the 311 LWC varies at this height.

The model can be used to find the distribution of sizes and velocities in a cloud of waveimpact sea spray. The droplet trajectories of the droplets, by considering the drag and body forces, 314 establish the paths and velocities of numerous droplets in the cloud of spray. The initial sizes are 315 based on the velocity-size dependence of the droplets, which was reported by Dehghani et al. 316 (2016a). The results of the numerical model will provide the dispersion of the droplets in front of 317 the vessel and the way the spray cloud travels over the deck. The model can be used to determine 318 the distributions of the sizes and velocities of the droplets in a cloud of spray. Using this model 319 can help marine icing researchers to gain a better understanding of the incoming water flux at 320 every point of a vessel. 


\section{Conclusions}

323 Distributions of the droplet sizes and velocities were obtained by using velocity-size dependence 324 of the droplets at the end of the breakup process and a droplet trajectory method. A vertical 325 distribution of sizes shows that the assumption of uniform sizes for the droplets would not be 326 accurate. The numerical results show that the smallest droplets, which are the high velocity 327 droplets, are slowed down by drag forces rapidly. The largest droplets, which are the low velocity 328 droplets, fall soon because of gravity. Therefore, medium-size droplets reach the highest 329 elevations. The distribution of the vertical velocities of the droplets shows that the upward droplets 330 change their movement to the downward direction after about $5 \mathrm{~m}$ traveling over the deck. The 331 maximum velocity increases as the spray cloud moves on the vessel. The horizontal drag force is 332 maximum at the stage of formation of the spray cloud. Drag forces change the droplet movement 333 directions. Body forces are dominant forces in the vertical direction. The droplets are affected by 334 the body forces and fall soon. Numerical results show that the maximum impingement height, 335 predicted by the model, is aligned with the field observations reported by Sharpov (1971). The

336 LWCs obtained by the numerical solutions are well aligned with the field observations reported 337 by Borisenkov et al. (1975). The new model provides a useful method for estimating the size and 338 velocity distribution in a cloud of spray.

340 Acknowledgments

341 The authors gratefully acknowledge the financial support from Statoil (Norway), MITACS, and 342 Petroleum Research Newfoundland and Labrador (PRNL) for this research. 


\section{4}

345

346

347

348

\section{References}

Borisenkov, Y.P., Zablockiy, G.A., Makshtas, A.P., Migulin, A.I., Panov, V.V., 1975. On the approximation of the spray cloud dimensions. Arkticheskii I Antarkticheskii Nauchno-

Issledovatelskii Instit. Trudy Leningrad: Gidrometeoizda (in Russia), pp. 121-126.

Bullock, G.N., Obhrai, C., Peregrine, D.H., Bredmose, H., 2007. Violent breaking wave impacts.

Part 1: Results from large-scale regular wave tests on vertical and sloping walls. Coastal Engineering, 54, 602-617.

Chung, K.K., Lozowski, E.P., 1998. A Three-Dimensional Time-Dependent Icing Model for a Stern Trawler. Journal of Ship research, 42(4), 266-273.

Chung, K.K., Lozowski, E.P., Zakrazewski, W.P., Gagnon, R., Thompson, T., 1998. Spraying experiments with a model stern traveler. Journal of Ship research, 42(4), 260-265.

Dehghani, S.R., Muzychka, Y.S., Naterer, G.F., 2016a. Droplet Trajectories of Wave-Impact Sea Spray on a Marine Vessel. Cold Region Science and Technology, (Accepted).

Dehghani, S.R., Naterer, G.F., Muzychka, Y.S., 2016b. Phase Change Heat Transfer in BrineSpongy Ice. Cold Region Science and Technology, (under review).

Dehghani, S.R., Saidi, M.H., Mozafari, A.A., Ghafourian, A., 2009. Particle Trajectory in a Bidirectional Vortex Flow. Particulate Science and Technology, 27, 16-34.

Dehghani, S.R., Saidi, M.H., Mozafari, A.A., Soleimani, F., 2013. Particle Dispersion Dependency on the Entrance Position in Bidirectional Flow. Particulate Science and Technology, 31, 576-584.

Forest, T.W., Lozowski, E.P., Gagnon, R., 2005. Estimating Marine Icing on Offshore Structures using RIGICE04. IWAIS XI, Montréal, June 2005. 
Galiev, S.U., Flay, R.G.J., 2014. Interaction of breaking waves with plates: The effect of hull

367 cavitation. Ocean Engineering, 88, 27-33.

368 Greco, M., Colicchio, G., Lugni, C., Faltinsen, O.M., 2013. 3D domain decomposition for 369 violent wave-ship interaction. International Journal for numerical methods in engineering, 95, $370 \quad 661-684$.

371 Gu, H.B., Qiana, L., Causona, D.M., Minghama, C.G., Lin, P., 2014. Numerical simulation of 372 water impact of solid bodies with vertical and oblique entries. Ocean Engineering. 75, 128-137.

373 Horjen, I., 2013 Numerical modeling of two-dimensional sea spray icing on vessel-mounted 374 cylinders. Cold Regions Science and Technology, 93, 20-35.

375 Horjen, I., 2015. Offshore drilling rig ice accretion modeling including a surficial brine film.

376 Cold Regions Science and Technology, 119, 84-110.

377 Kulyakhtin, A., Tsarau, A., 2014. A time-dependent model of marine icing with application of 378 computational fluid dynamics. Cold Regions Science and Technology, 104-105, 33-44.

379 Lozowski, E.P., Szilder, K., Makkonen, L., 2000. Computer simulation of marine ice accretion. 380 Philosophical Transactions of the Royal Society, London, A, 358, 2811-2845.

381 Panov, V.V., 1978. Icing of Ships. Polar Geography, 2 (3), 166-186.

382 Ren. N., Marshall, A.W., 2014. Characterizing the initial spray from large Weber number 383 impinging jets. International Journal of Multiphase Flow, 58, 205-213.

384 Ryerson, C.C., 1995. Superstructure spray and ice accretion on a large U.S. Coast Guard cutter. 385 Atmospheric Research, 36 (3-4), 321-337.

386 Sharpov, A.V., 1971. On the intensity of superstructure icing of small vessels (MFV type). In 387 Theoretical and Experimental Investigations of the Conditions of Ship Icing, 95-97. 
388 Shipilova, O., Kulyakhtin, A., Tsarau, A., Libby, B., Moslet, P.O., Loset, S., 2012. Mechanism 389 and Dynamics of Marine Ice Accretion on Vessel Archetypes, OTC-23762-MS, OTC Arctic

390 Technology Conference, December, Houston, Texas, USA.

391 Zakrzewski, W.P., 1986. ICING OF SHIPS. PART I: SPLASHING A SHIP WITH SPRAY.

392 NOAA Technical Memorandum ERL PMEL-66.

393 Zakrzewski, W.P., 1987. Splashing a ship with collision-generated spray. Cold Regions Science 394 and Technology, 14 (1), 65-83.

395 Zakrzewski, W.P., Lozowski, E.P., 1988. Estimating the extent of the spraying zone on a sea396 going ship. Ocean Engineering, 15(5), 413-429.

397

398

399

400

401

402

403

404

405

406

407

408

409

410 
Table 1. Comparison between numerical results, field observations, and previous data

\begin{tabular}{lcrr}
\hline Positions on the Vessel & \multicolumn{3}{c}{ Results } \\
\cline { 2 - 4 } & Lozrazowski and & Sharpov & Numerical Results \\
& $5.85 \mathrm{~m}$ & 5.5 to $7.9 \mathrm{~m}$ & $6.28 \mathrm{~m}$ \\
Front side of the structure & $2.07 \mathrm{~m}$ & Spray hits & $2.33 \mathrm{~m}$ \\
Roof of the structure & No spray & No spray & No spray \\
Boat deck & No spray & No spray & No spray \\
Entire vessel sprayed & No & No & No \\
& & & \\
\hline
\end{tabular}

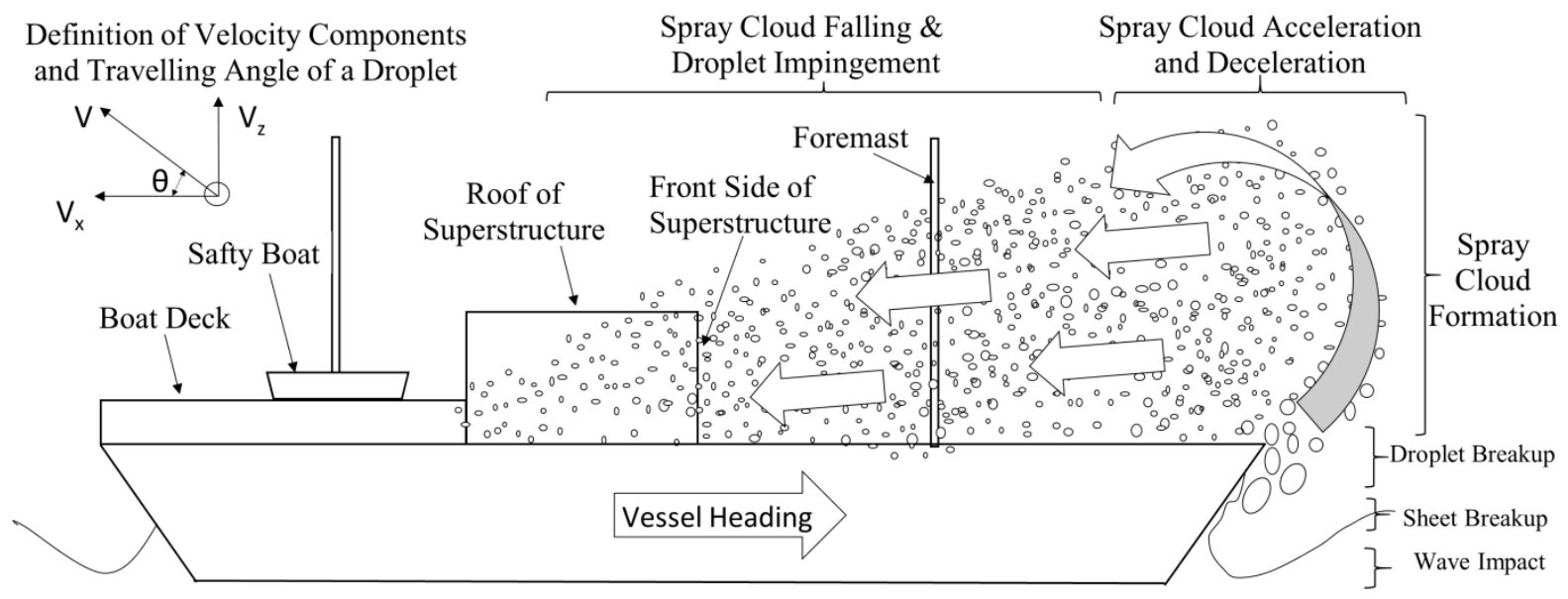

Fig. 1. Schematic of the development stages of wave-impact sea spray from creation to 


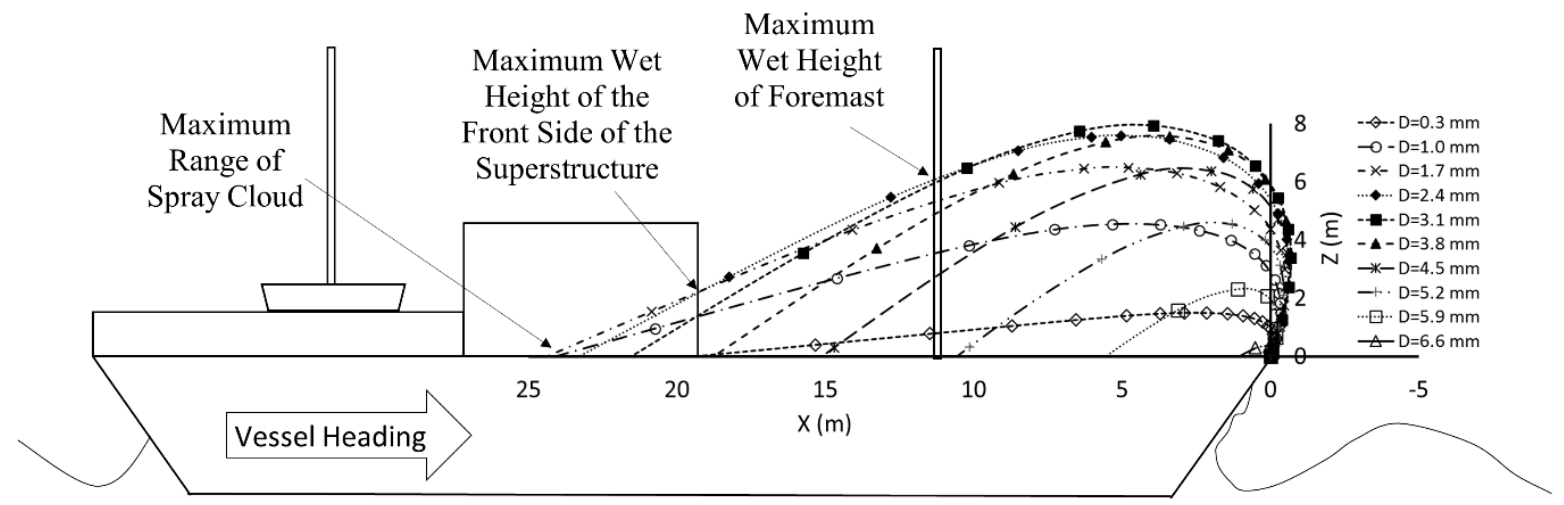

Fig. 2. Droplet trajectories of the spray cloud over the MFV and the maximum wet heights and

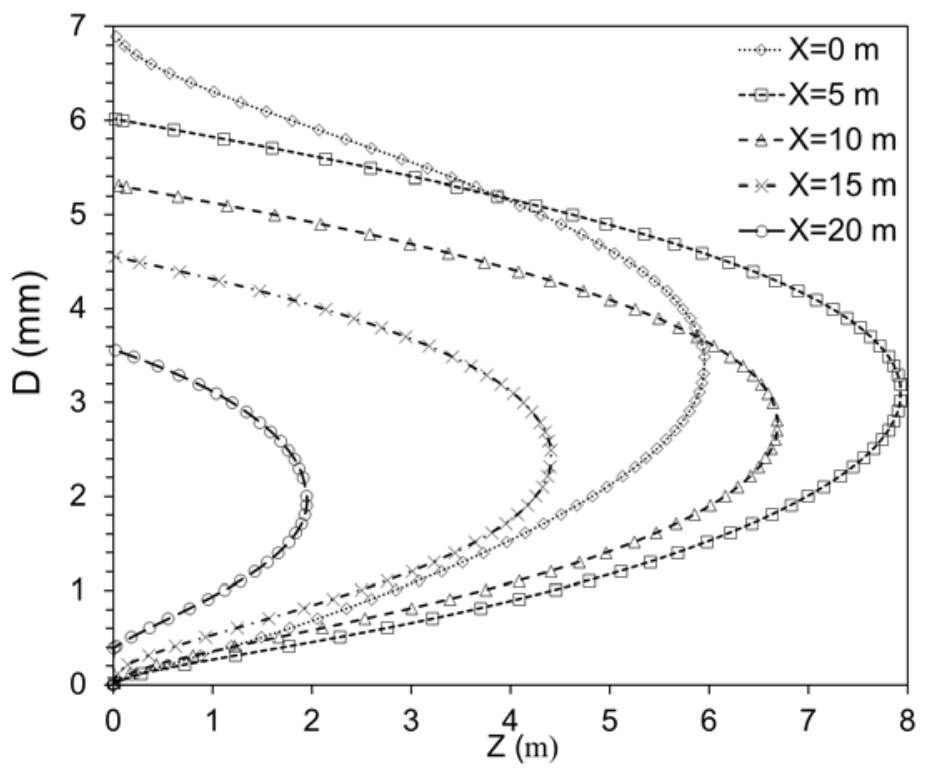




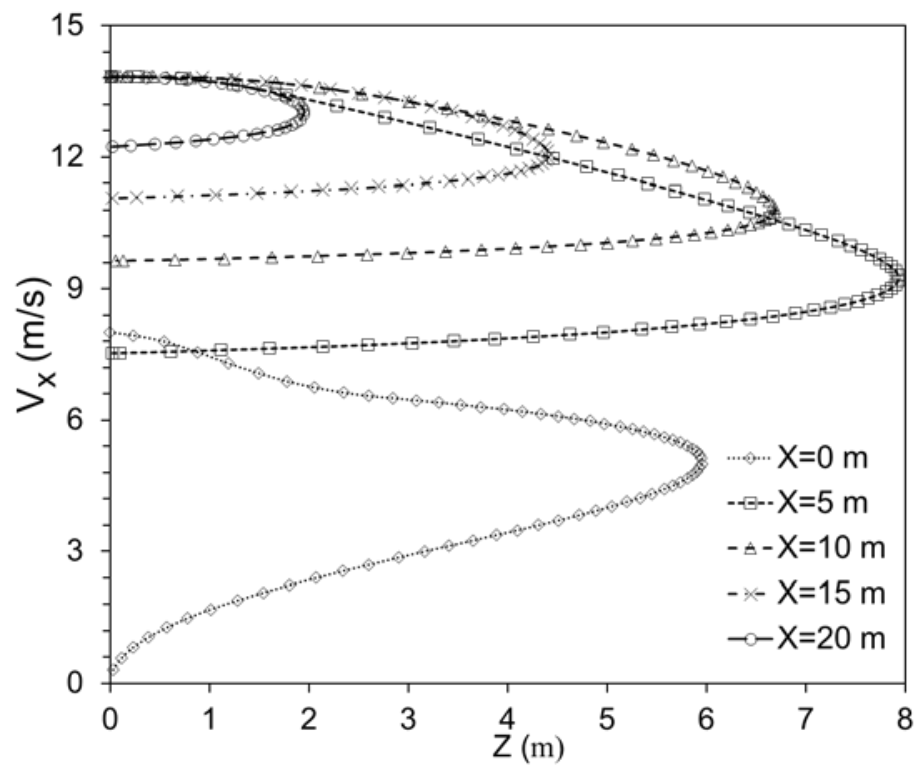

431 Fig. 4. Vertical distribution of the horizontal components of the droplet velocities in a spray cloud

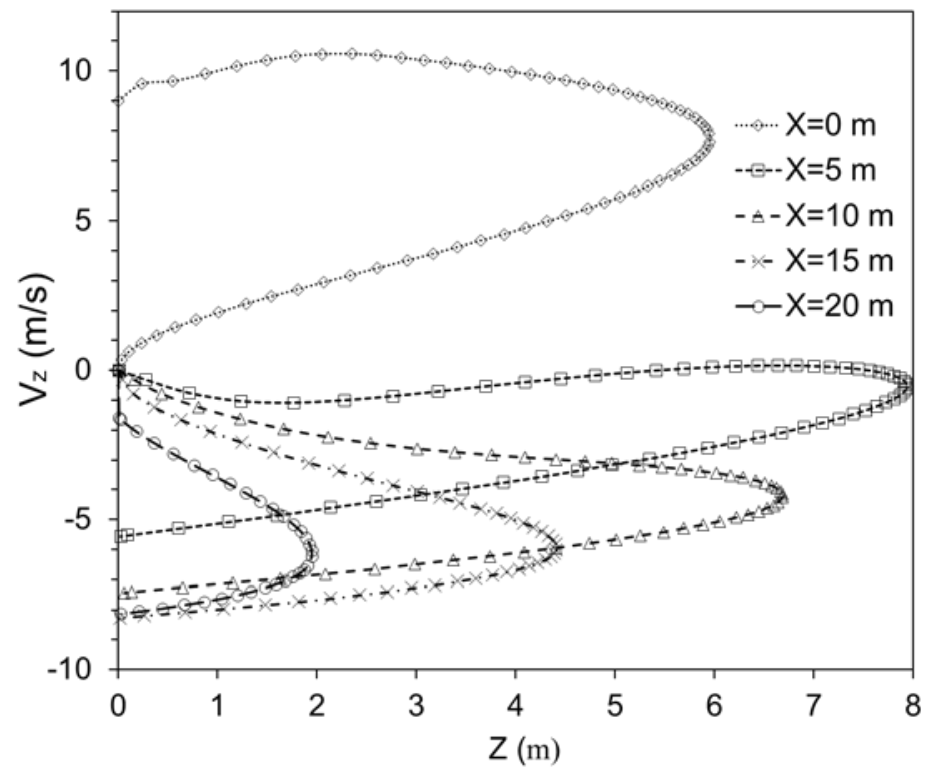

435 Fig. 5. Vertical distributions of the vertical components of the droplet velocities in a spray cloud 


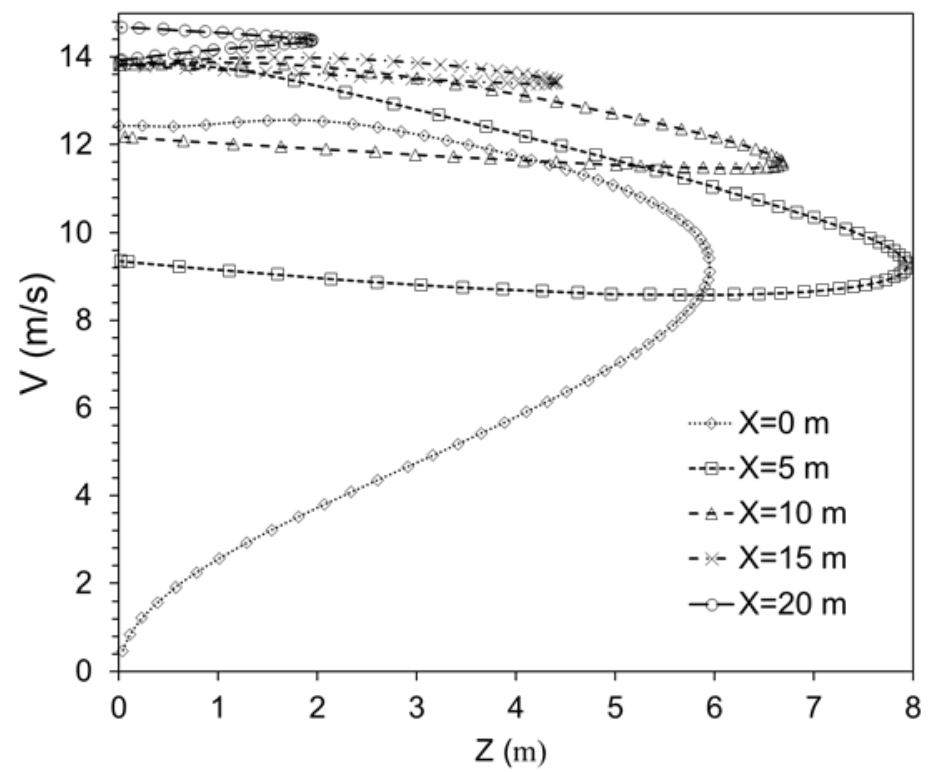

440

Fig. 6. Vertical distribution of the droplet velocities in a wave-impact sea spray

441

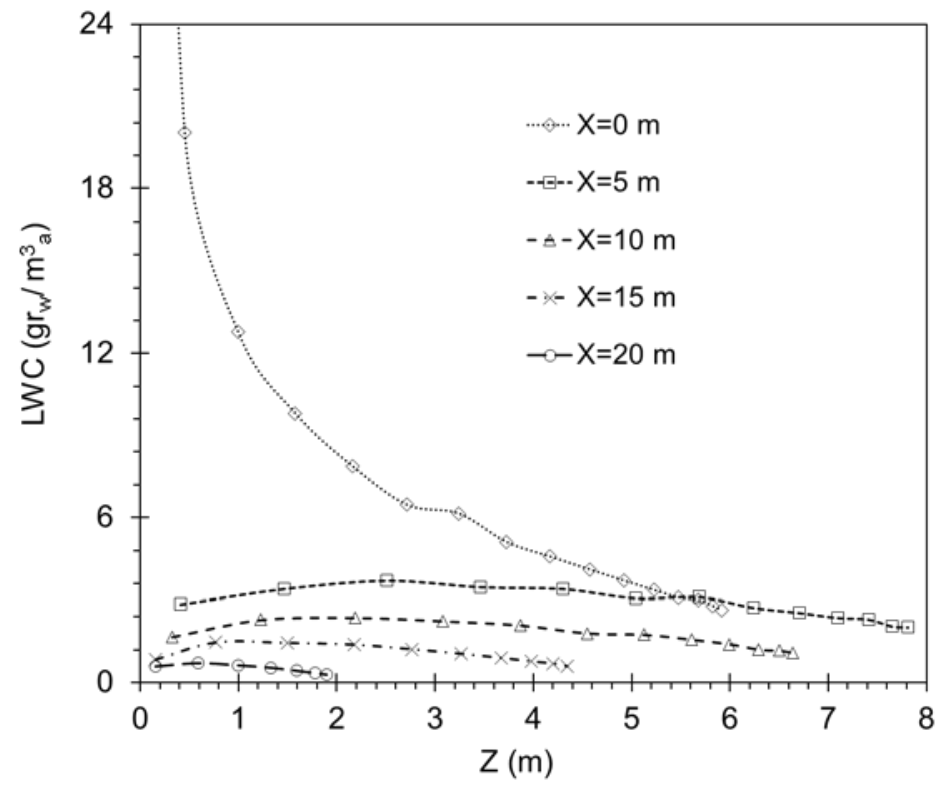

Fig. 7. Variations of the LWC at various distances from the bow 


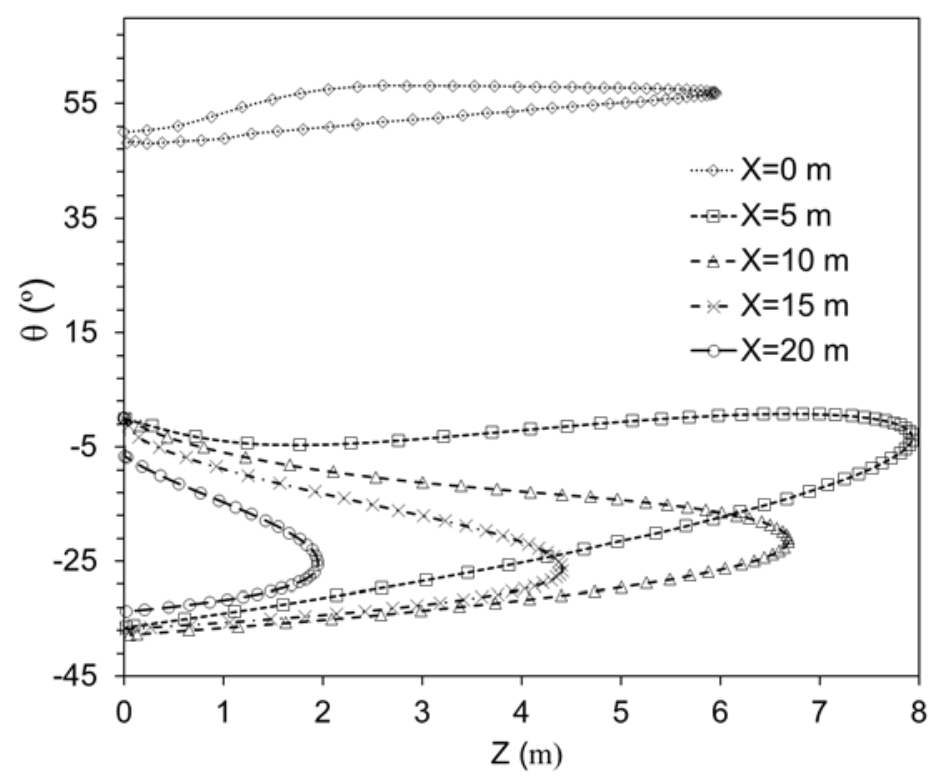

Fig. 8. Vertical distribution of the traveling angles of the droplets in a spray cloud

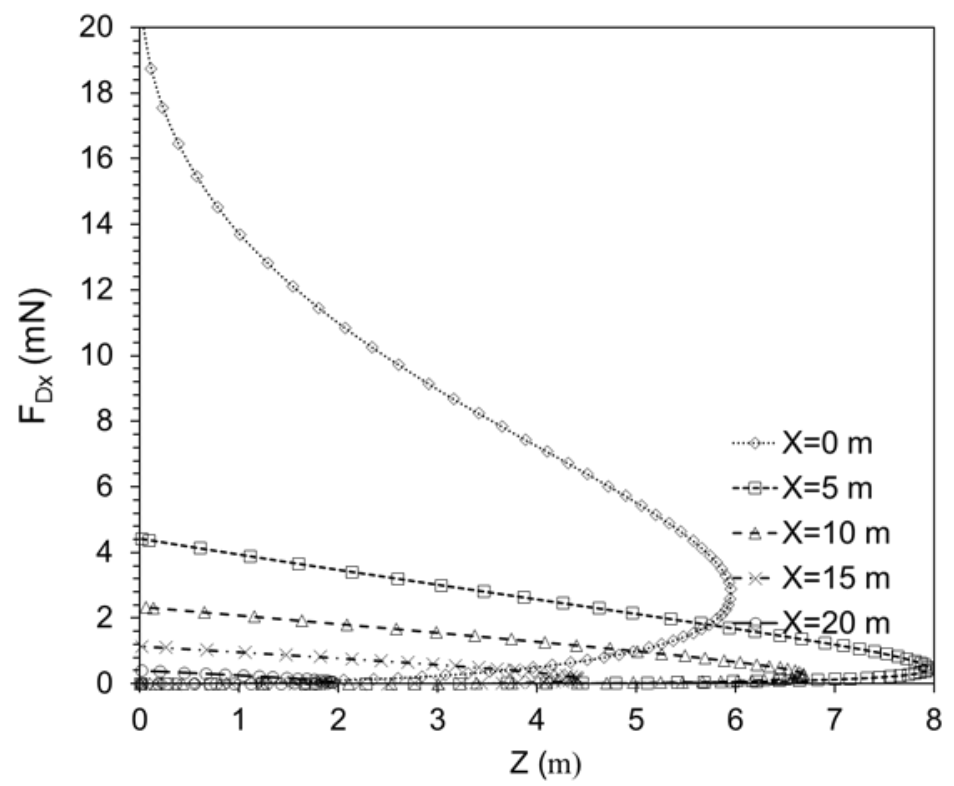

Fig. 9. Vertical distributions of the horizontal components of drag forces exerted on the droplets 


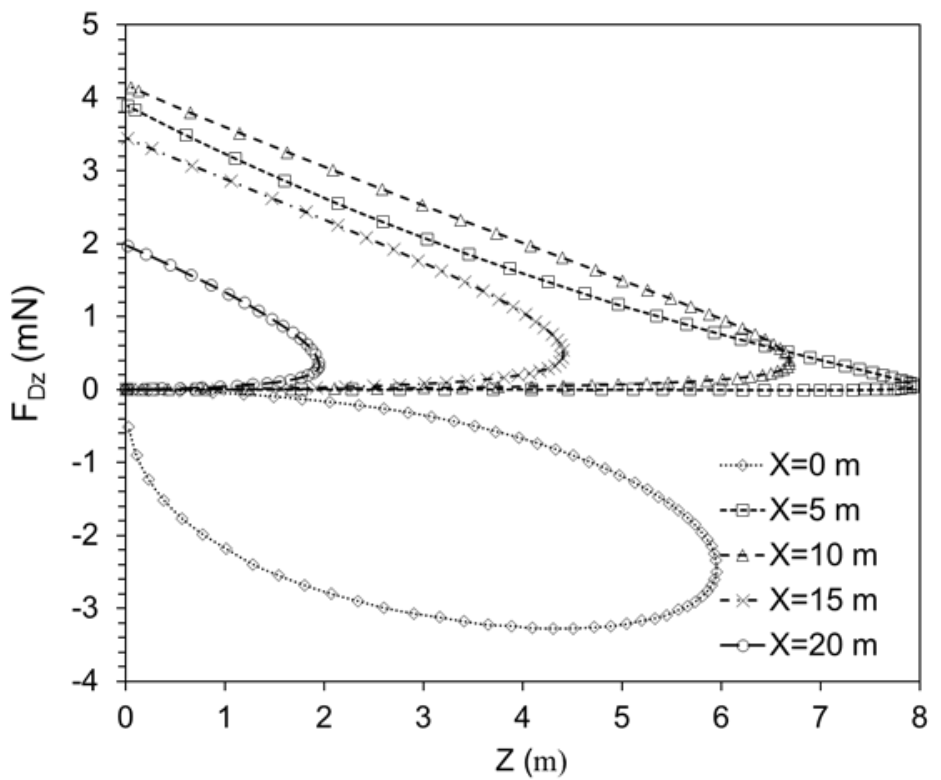

Fig. 10. Vertical distribution of vertical components of drag forces exerted on droplets

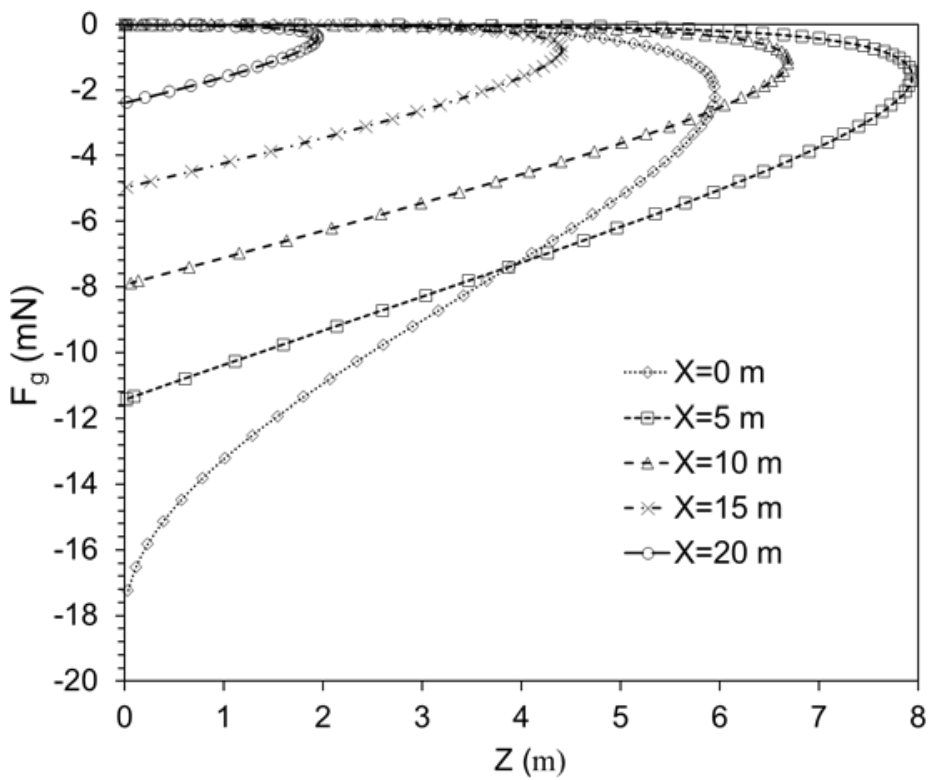




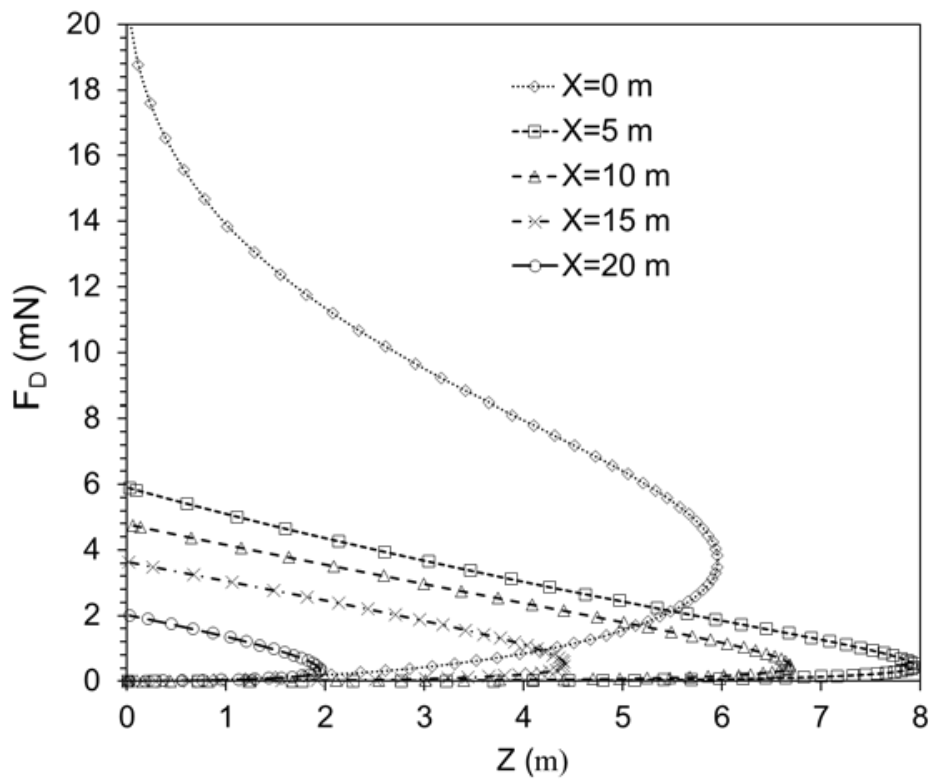

464 Fig. 12. Vertical distributions of total drag forces exerted on the droplets travelling on the MFV

465

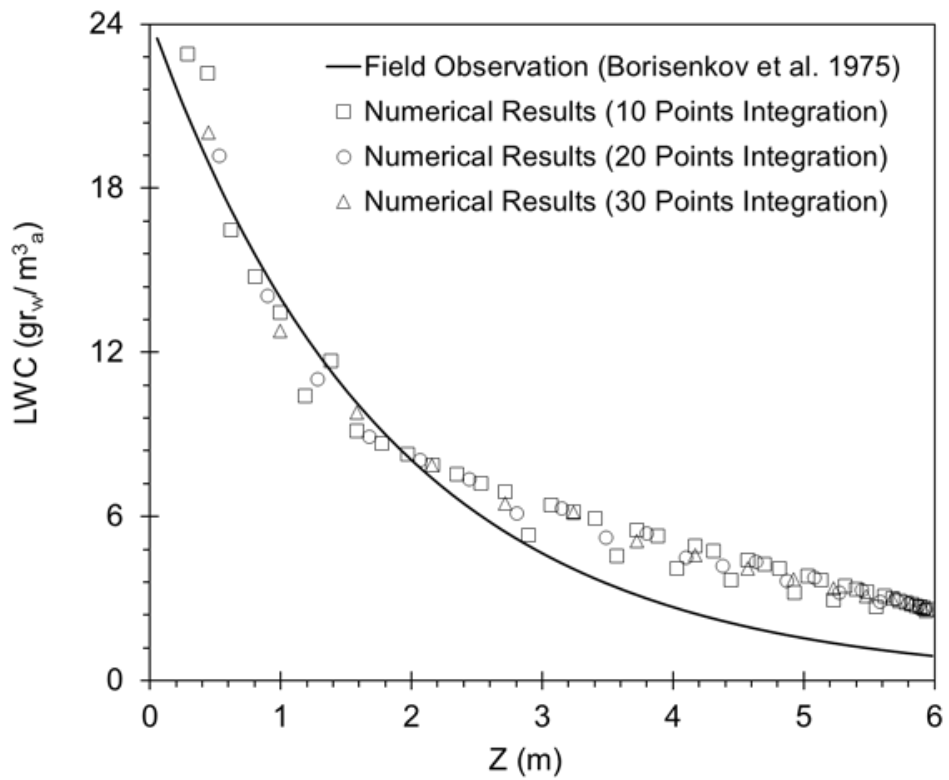

Fig. 13. Comparison between numerical results and field observations 